\title{
MicroRNA-29c Increases the Chemosensitivity of Pancreatic Cancer Cells by Inhibiting USP22 Mediated Autophagy
}

\author{
Limin Huang ${ }^{a}$ Chaoquan $\mathrm{Hu}^{b}$ Hui Cao ${ }^{a}$ Xiaoliang Wu ${ }^{a}$ Rongpin Wang \\ He Luc Hong Lid Hui Chen ${ }^{\mathrm{a}}$ \\ ${ }^{a}$ Department of Oncology, Guizhou Provincial People's Hospital, Guiyang, Guizhou, b Department of \\ Surgery, Affiliated Hospital, GuiZhou Medical University, Guiyang, China, INSERM UMR_S 1165/Paris 7, \\ Hôpital Saint Louis, Paris, dINSERM U1234, Rouen University, Rouen, France
}

\author{
Key Words \\ Pancreatic cancer • MiR-29c • USP22 • Autophagy • Chemosensitivity
}

\begin{abstract}
Background/Aims: Pancreatic cancer (PC) is an aggressive malignancy with a poor survival rate. Despite advances in the treatment of $P C$, the efficacy of therapy is limited by the development of chemoresistance. Here, we examined the role of microRNA-29c (miR-29c) and the involvement of autophagy and apoptosis in the chemoresistance of PC cells in vivo and in vitro. Methods: We employed qRT-PCR, western blot and immunofluorescence to examine the expression level of miR-29c, USP22 and autophagy relative protein. In addition, we used MTT assay to detect cell proliferation and transwell assay to measure migration and invasiveness. The apoptosis was determined using annexin V-FITC/PI apoptosis detection kit by flow cytometry. Luciferase reporter assays confirmed the relationship between USP22 and miR-29c. Results: miR-29c overexpression in the PC cell line PANC-1 enhanced the effect of gemcitabine on decreasing cell viability and inducing apoptosis and inhibited autophagy, as shown by western blotting, immunofluorescence staining, colony formation assays, and flow cytometry. Ubiquitin specific peptidase (USP)-22, a deubiquitinating enzyme known to induce autophagy and promote PC cell survival, was identified as a direct target of miR-29c. USP22 knockdown experiments indicated that USP22 suppresses gemcitabine-induced apoptosis by promoting autophagy, thereby increasing the chemoresistance of PC cells. Luciferase reporter assays confirmed that USP22 is a direct target of miR-29c. A xenograft mouse model demonstrated that miR-29c increases the chemosensitivity of PC in vivo by downregulating USP22, leading to the inhibition of autophagy and induction of apoptosis. Conclusions: Taken together, these findings reveal a potential mechanism underlying the chemoresistance of PC cells mediated by the regulation of USP22-mediated autophagy by miR-29c, suggesting potential targets and therapeutic strategies in PC.

L. Huang and C. Hu contributed equally to this work.

\section{KARGER}




\section{Cellular Physiology Cell Physiol Biochem 2018;47:747-758

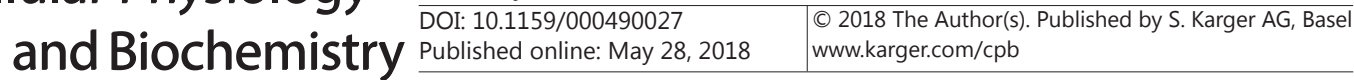 \\ Huang et al.: Inhibition of USP22-Mediated Autophagy by MIR-29c Increases Chemosensitivity}

\section{Introduction}

Pancreatic cancer (PC) is an aggressive malignancy and the fourth leading cause of cancer-related death in Western countries [1]. The 5-year survival rate of PC is approximately 7\%; the high mortality of PC is mainly associated with the high potential of pancreatic cancer cells for invasion and metastasis and the fact that approximately $80 \%$ of patients have metastatic disease at diagnosis [2-4]. In the early stages, PC is treated by surgical resection and adjuvant chemotherapy $[5,6]$. In patients with advanced PC, although several chemotherapy combinations are currently in clinical trials, gemcitabine remains the first line therapy, and PC cells show higher susceptibility to gemcitabine than to other anticancer drugs $[7,8]$. However, most patients develop chemoresistance, which remains an important limitation in the treatment of this disease. Chemoresistance is a multifactorial and complex process, and several mechanism of resistance to gemcitabine have been proposed. Nevertheless, improving our understanding of the pathways involved is an important step towards identifying effective therapies.

MicroRNAs (miRNAs) are a group of evolutionarily conserved small noncoding RNA molecules that negatively modulate gene expression by binding to the $3^{\prime}$ untranslated region (3'-UTR) of target mRNAs leading to mRNA degradation or suppression of translation [9]. miRNAs can function as tumor suppressors or oncogenes according to their target mRNAs, and deregulation of miRNA expression is associated with many pathologies including cancer [10-14]. Several miRNAs have been identified that are associated with PC, including miR200c, miR-375, miR-10b, miR-181b, miR-196a, miR-21, and miR-29c [15-20]. Silencing or downregulation of miR-29c has been reported in many cancers including hepatocellular carcinoma, nasopharyngeal cancer, glioma, gastric cancer, bladder cancer, and colorectal cancer [21-26]. However, miR-29c is overexpressed in osteosarcoma [21].

Ubiquitin-specific protease 22 (USP22) is a deubiquitinating enzyme involved in the regulation of several cellular processes, including growth, differentiation, cell cycle progression, transcriptional activation, and signal transduction [27, 28]. Alterations in USP22 expression are associated with poor prognosis in patients with invasive breast cancer, colorectal cancer, and papillary thyroid carcinoma, and USP22 is therefore considered as a marker of metastasis and poor prognosis in cancer [29-34]. USP22 was shown to induce autophagy and promote the survival of Panc- 1 cells, suggesting an oncogenic role of USP22 in PC [35]. Autophagy is a self-degradation process characterized by the formation of the autophagosome, a double-membrane-bound vacuole that engulfs cellular material targeted for degradation and fuses with the lysosome, leading to the degradation of its contents [36]. Beclin-1, an interacting partner of the antiapoptotic protein Bcl-2, is an autophagyrelated protein that plays a role in the formation of autophagosomes, which also involves the cytosolic form of microtubule-associated protein 1 light chain 3 (LC3-I). LC3-I is conjugated to phosphatidylethanolamine to generate LC3-II, which is recruited to the autophagosomal membrane and degraded after the fusion of autophagosomes to lysosomes [37]; therefore, the LC3-II/LC3-I ratio is a measure of autophagic activity. Autophagy promotes cancer cell survival under specific conditions, and inhibition of autophagy increases the chemosensitivity of cancer cells $[38,39]$.

In the present study we examined the role of miR-29c and USP22 in the chemoresistance of PC cells in vitro and in vivo and explored the underlying mechanisms. Our results indicated that miR-29c increased the chemosensitivity of PC by inducing apoptosis and inhibiting autophagy. USP22 knockdown inhibited cell proliferation and promoted apoptosis by inducing autophagy. We showed that miR-29c downregulated USP22 and inhibited USP22mediated autophagy, decreasing the chemoresistance of $\mathrm{PC}$ in vitro and in vivo. These results indicate that the overexpression of USP22 may promote autophagy and the oncogenic potential of PC cells. 


\section{Cellular Physiology Cell Physiol Biochem 2018;47:747-758 \begin{tabular}{ll|l} 
and Biochemistry & Dublished online: May 28, 2018 & $\begin{array}{l}\text { @ 2018 The Author(s). Published by S. Karger AG, Basel } \\
\text { www.karger.com/cpb }\end{array}$ \\
\hline
\end{tabular} Huang et al.: Inhibition of USP22-Mediated Autophagy by MIR-29c Increases Chemosensitivity}

\section{Materials and Methods}

\section{Ethics statement}

Nude mice (BALB/c-nu) were purchased from the SPF Laboratory Animal Center at Shanghai Research Center for Model Organisms. All animal procedures were in accordance with institutional guidelines and experiments were performed under approval from the Animal Care Committee of the Guizhou Province People's Hospital (Guizhou, China).

\section{Cell culture, reagents and antibodies}

The human pancreatic cancer cell line PANC-1 was purchased from the American Type Culture Collection (ATCC; Danvers, MA, USA). The cell lines were maintained in RPMI 1640 (Hyclone, SH30027.02) with 10\% fetal bovine serum (Invitrogen Carlsbad, CA, USA). Anti-USP22, Beclin-1, caspase-3, Bcl-2, Bax, LC3, P62, and GAPDH antibodies were purchased from Santa Cruz (Santa Cruz Biotechnology, Santa Cruz, $\mathrm{CA}$ ). Annexin V (AV) and propidium iodide (PI) were purchased from Sigma (Sigma-Aldrich, St. Louis, MO, USA).

\section{Measurement of cell viability}

The growth or viability of cells was assessed with the MTT reagent (Sigma-Aldrich, M2003). Cells were seeded into 96-well plates at a density of $4 \times 10^{3}$ cells/well. After the indicated treatments, MTT was added to each well (final concentration of $0.5 \mathrm{mg} / \mathrm{mL}$ ) followed by incubation at $37^{\circ} \mathrm{C}$ for $4 \mathrm{~h}$. The supernatant was removed, and DMSO was added to dissolve the blue-purple formazan crystals. The optical density of the samples was measured at a wavelength of $540 \mathrm{~nm}$ using a spectrophotometer (Thermo Scientific, Multiskan EX).

\section{Apoptosis detection}

To measure the rate of apoptosis, PANC-1 cells in the different treatment groups were collected and washed with PBS. Apoptotic cells were differentiated from viable or necrotic cells by the combined application of Annexin V (AV)-FITC and propidium iodide (PI). Cells were washed twice and adjusted to a concentration of $1 \times 10^{6}$ cells $/ \mathrm{mL}$ with cold D-Hanks buffer. Then, AV-FITC $(10 \mu \mathrm{L})$ and PI $(10 \mu \mathrm{L})$ were added to $100 \mu \mathrm{L}$ of cell suspension and incubated for $15 \mathrm{~min}$ at room temperature in the dark. Finally, 400 $\mu \mathrm{L}$ of binding buffer was added to each sample without washing and analyzed by flow cytometry. Each experiment was performed at least in triplicate.

\section{Autophagy assays}

For assessment of the effect of miR-29c on autophagy, $1 \times 10^{5} \mathrm{PC}$ cells transfected with the GFP-LC3B vector (Invitrogen) were plated in 12 well plates and grown at $37^{\circ} \mathrm{C}$ for $24 \mathrm{~h}$. Cells were then transfected with $10 \mathrm{nM}$ control or miR-29c mimics using DharmaFECT $® 1$. At $24 \mathrm{~h}$ post-transfection, cells were pretreated with or without $0.1 \mathrm{mM}$ rapamycin in complete media for $6 \mathrm{~h}$ and then exposed to gemcitabine $(100 \mathrm{ng} / \mathrm{ml})$ for $24 \mathrm{~h}$. Subsequently, total proteins were harvested and subjected to western blotting or immunofluorescence analysis.

\section{Soft agar assays}

PANC- 1 cells were plated at a density of $3 \times 10^{5}$ cells per well in 6 well plates and grown at $37^{\circ} \mathrm{C}$ for 24 h. Cells were then transfected with $20 \mathrm{nM}$ mimic control or miR-29a mimic. PANC-1 cells transfected with control or miR-29a mimics were plated at a density of $1.5 \times 10^{3}$ cells per well in a 6 well plates containing $0.5 \%$ top agarose and $1 \%$ bottom agarose (BioRad, 162-0137). After 20 days, colonies were stained with crystal violet and positive colonies were counted under low power bright field microscopy.

\section{Western blot analysis}

The harvested cells were lysed on ice for $30 \mathrm{~min}$ with lysis buffer (Cell Signaling Technology, 9803). Lysates were cleared by centrifugation at $14,000 \mathrm{rpm}$ for $10 \mathrm{~min}$ at $4{ }^{\circ} \mathrm{C}$. Protein concentration was measured using the Bradford assay (Bio-Rad Laboratories, 500-0006). For western blot analysis, cell lysates were boiled for 5 min with sample buffer and separated by SDS-polyacrylamide gel electrophoresis. The proteins were transferred to PVDF membranes (Millipore, IPVH00010), which were blocked with 5\% skim 


\section{Cellular Physiology Cell Physiol Biochem 2018;47:747-758

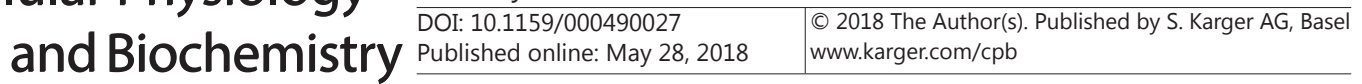 Huang et al.: Inhibition of USP22-Mediated Autophagy by MIR-29c Increases Chemosensitivity}

milk in TBST buffer (20 mM Tris-HCl, pH 7.4, $150 \mathrm{mM} \mathrm{NaCl,} 0.1 \%$ Tween 20) for 1 hour and then stained with primary antibodies at $4^{\circ} \mathrm{C}$ overnight followed by incubation with secondary antibodies. The binding of each antibody was detected using an enhanced chemiluminescence kit (PerkinElmer, NEL105001EA). The signals were detected by X-ray films (Fuji, 47410 08399) or a UV transilluminator (UVP Ltd., BioSpectrum ${ }^{\mathrm{TM}}$ 500 Imaging System) and analyzed by the Gel-Pro Analyzer 4.0 software (Media Cybernetics).

Small interfering RNA (siRNA) transfection

Control siRNA and siRNA specifically targeted against USP22 (5'-GCAAGGCCAAGTCCTGTATCT-3') and negative control SiRNA (5'-UUCUCCGAACGUGUCACGUTT-3') were purchased from RiboBio Co., Ltd. (Guangzhou, China). Cells were plated in 6-well plates and grown to $80 \%$ confluence before transient transfection with siRNAs (100 pmol per well) using Lipofectamine ${ }^{\circledR} 2000$ (Invitrogen; Thermo Fisher Scientific), according to the manufacturer's instructions. After $48 \mathrm{~h}$ of transfection, cells were collected for other experiment.

\section{Luciferase reporter assay}

To construct luciferase reporter vectors, the 3'-UTR of USP22 containing the predicted potential miR29c binding sites was amplified by PCR and subcloned downstream of the luciferase gene into the PYrMirTarget luciferase vector (Ambion, Inc., Austin, TX, USA). The 3'-UTR of USP22 was amplified from a cDNA library with the following primers:

forward: $\quad 5$-'-CTCGAGCCTCTGCACCTGGGACCCATCGGGTCGGG-3' and reverse: 5'-GCGGCCGCCCGAGTGCTGGGGAACGCC-3'. The mutant 3'-UTR of USP22 (in which nine nucleotides were mutated in the binding sites) was amplified using the following primer sequences:

forward: 5'-CTCGAGCCTCTGCCACTGCCACCCTAGGCCTCGGG-3' and reverse: 5'-GCGGCCGCCCGAGTGCTGGGGAACGCC-3'.

For luciferase assays, cells were cultured in 24-well plates and co-transfected with $50 \mathrm{ng}$ of the corresponding vectors containing firefly luciferase together with $25 \mathrm{ng}$ of miR-29c or control. Transfection was performed using Lipofectamine 2000 (Invitrogen). At $48 \mathrm{~h}$ post-transfection, relative luciferase activity was calculated by normalizing the Firefly luminescence to the Renilla luminescence using a Dual-Luciferase Reporter Assay (Promega, Madison, WI, USA) according to the manufacturer's instructions.

\section{Mouse xenograft model}

Equal numbers of cells $\left(2 \times 10^{6}\right.$ cells $)$ were resuspended in $100 \mu \mathrm{L}$ of PBS and subcutaneously injected into 4-week-old nude mice. Tumor volume was calculated with the formula $V=1 / 2 a \times b^{2}$ (a, the longest tumor axis; $b$, the shortest tumor axis). When the volume of tumors reached $300 \mathrm{~mm}^{3}$, the mice received equal volumes of gemcitabine (50 mg/kg) or vehicle (PBS) via intraperitoneal injections once a week for 4 weeks. At the study end point, all mice were sacrificed and tumors were excised.

\section{RNA extraction and qRT-PCR}

Total RNA was extracted from tissues or cells using the Trizol reagent (Invitrogen) according to the manufacturer's instructions. Total RNA was eluted with RNase-free water and stored at $-80^{\circ} \mathrm{C}$. RNA concentrations were determined by Epoch spectrophotometry. Quantification of mature miR-29c and endogenous control U6 snRNA was performed using TaqMan assays 000409 and 001006 with the supplied assay-specific RT primers (Applied Biosystems, Foster City, CA, USA). Data were analyzed using the $2^{-\Delta \Delta C t}$ method.

\section{Statistical analysis}

Continuous variables are presented as the mean \pm standard deviation (SD). One-way ANOVA was carried out for multiple comparisons using GraphPad Prism 5.0 software (GraphPad Software, Inc., La Jolla, CA, USA). P-values $\leq 0.05$ indicate a statistically significant difference.

\section{Results}

miR-29c promotes the chemosensitivity of PC by inhibiting autophagy

To examine the role of miR-29c in the chemoresistance of PC cells, Panc-1 cells were transfected with miR-29c overexpression vector or miR-29c-NC and treated with or without 


\section{Cellular Physiology Cell Physiol Biochem 2018;47:747-758 \begin{tabular}{ll|l}
${ }$ and Biochemistry Published online: May 28, $2018 }$ & $\begin{array}{l}\text { ( ) 2018 The Author(s). Published by S. Karger AG, Basel } \\
\text { www.karger.com/cpb }\end{array}$ \\
\cline { 2 - 3 }
\end{tabular} \\ Huang et al.: Inhibition of USP22-Mediated Autophagy by MIR-29c Increases Chemosensitivity}

gemcitabine. Mimic transfection resulted in an approximately 3.5-fold upregulation of miR29c compared with its expression in untransfected and negative controls (Fig. 1A). miR-29c overexpression significantly increased the effect of gemcitabine on inhibiting cell growth and colony formation (Fig. 1B-D). Flow cytometric analysis showed that miR-29c overexpression significantly increased the effect of gemcitabine on inducing apoptosis, resulting in an approximately two-fold increase in the rate of apoptosis compared with the NC control (Fig. $1 \mathrm{E}$ and F). Western blot analysis showed that miR-29c mimic transfection enhanced the gemcitabine-induced upregulation of caspase-3, downregulation of $\mathrm{Bcl}-2$, and upregulation of Bax, suggesting that miR-29c increased gemcitabine-induced apoptosis in Panc-1 cells (Fig. $1 \mathrm{~L}-0$ ). Immunofluorescence staining for LC3 showed that miR-29c overexpression abolished the gemcitabine-induced formation of autophagosomes (Fig. 1G). This was confirmed by western blotting, which showed that miR-29c reversed the gemcitabine-induced increase in the LC3-II/LC3-1 ratio, the upregulation of Beclin-1, and downregulation of P62, suggesting that miR-29c inhibited gemcitabine-induced autophagy (Fig. 1H-K). These effects occurred

Fig. 1. miR-29c promotes the chemosensitivity of pancreatic cancer (PC) cells by inhibiting autophagy activation in vitro. Panc-1 cells were transfected with miR-29c overexpression mimics vector or miR-29c$\mathrm{NC}$ for $48 \mathrm{~h}$, followed by treatment with $100 \mathrm{ng} / \mathrm{ml}$ gemcitabine for $24 \mathrm{~h}$. (A) The expression of miR-29c was measured by qRt-PCR in cells transfected with miR-29c mimics or miR-29c-NC. Data are presented as the mean $\pm \mathrm{SD}$. ${ }^{* * *} \mathrm{p}<0.001$ versus the control. (B) Cell viability was measured by MTT assay. Data are presented as the mean \pm SD. ${ }^{* * *} \mathrm{p}<0.001$ versus the control. ${ }^{\# \# \# p}$ $<0.001$ versus gemcitabine-treatment control group. (C and D) Cell proliferation was examined by colony formation assay. Data are presented as the mean \pm SD. ${ }^{* * *} \mathrm{p}<0.001$ versus the control. $\# \# \#<0.001$ versus gemcitabine-treatment control group. ( $\mathrm{E}$ and F) Apoptotic cells were detected using Annexin V-PI double staining. Data are presented as the mean $\pm \mathrm{SD}$. ${ }^{* * *} \mathrm{p}<0.001$ versus the control. ${ }^{\# \#} \mathrm{p}<0.001$ versus gemcitabinetreatment control group. (G) Representative images of immunofluorescence staining for LC3 in PC cells. (H-I) Western blot analysis of autophagy (H-K) and apoptosis (L-O) related proteins. Data are presented as the mean \pm SD. ${ }^{* * *} \mathrm{p}<0.001$ versus the control. $\# \#$ p $<0.001$ versus gemcitabine-treatment control group. (P and Q) Western blot analysis of USP22 expression. Data are presented as the mean $\pm \mathrm{SD} .{ }^{* *} \mathrm{p}<0.01,{ }^{* * *} \mathrm{p}<0.001$

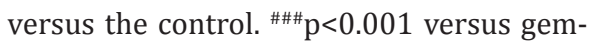
citabine-treatment control group.

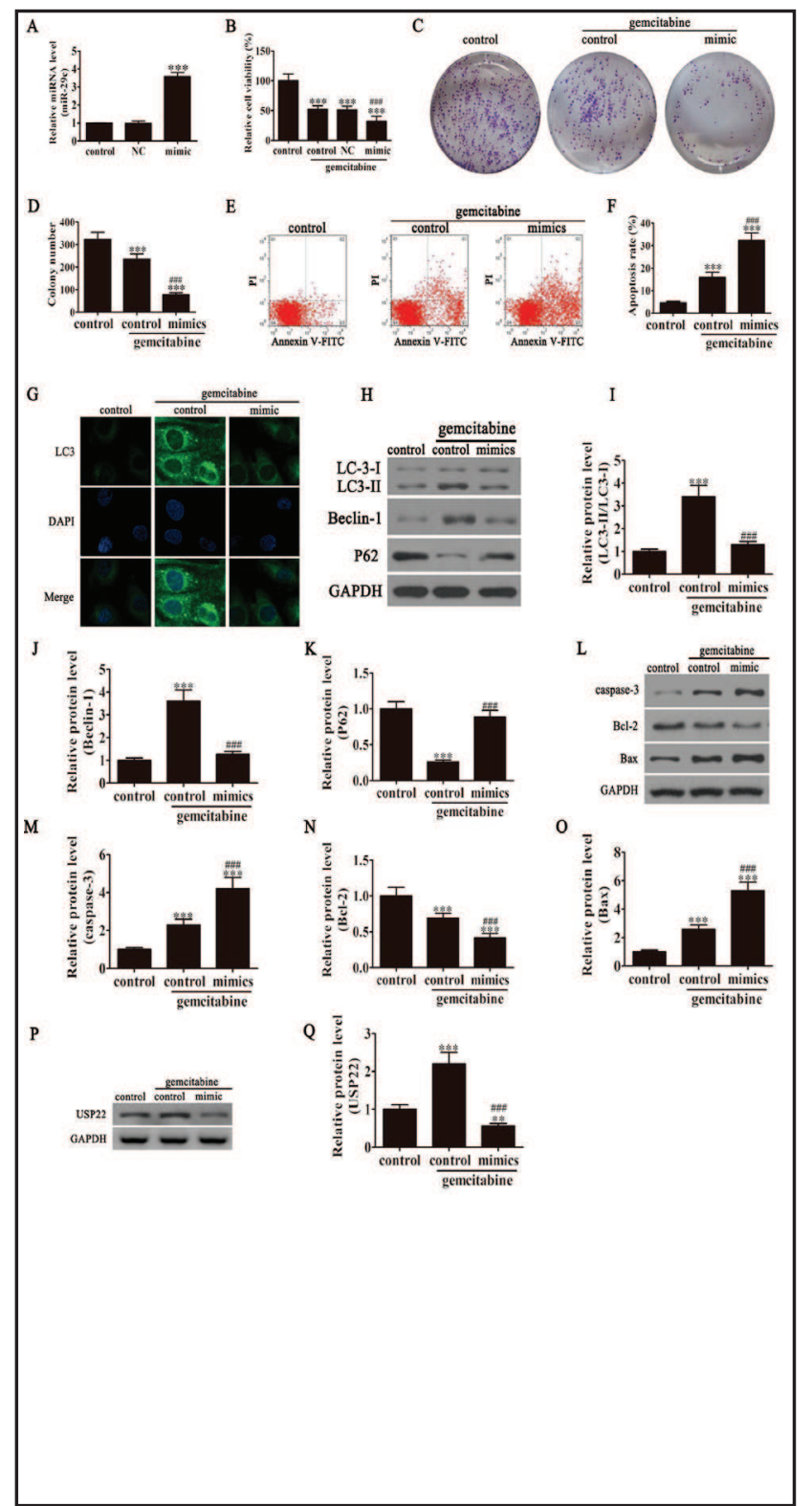




\section{Cellular Physiology Cell Physiol Biochem 2018;47:747-758 \begin{tabular}{ll|l} 
and Biochemistry $10.1159 / 000490027$ & $\begin{array}{l}\text { D 2018 The Author(s). Published by S. Karger AG, Base } \\
\text { www.karger.com/cpb }\end{array}$ \\
\cline { 2 - 3 }
\end{tabular} \\ Huang et al:: Inhibition of USP22-Mediated Autophagy by MIR-29c Increases Chemosensitivity}

Fig. 2. USP22 knockdown inhibits growth and promotes apoptosis in PC cells by increasing autophagy activation. Panc-1 cells were transfected with siRNA against USP22 for $48 \mathrm{~h}$, followed by treatment with $100 \mathrm{ng} / \mathrm{ml}$ gemcitabine for $24 \mathrm{~h}$. (A and B) The expression of USP22 was measured by qRt-PCR (A) and western blotting (B). Data are presented as the mean \pm SD. ${ }^{* * *} \mathrm{p}<0.001$ versus the control. (C) Cell viability was measured by the MTT assay. Data are presented as the mean $\pm \mathrm{SD}$. ${ }^{* * *} \mathrm{p}<0.001$ versus the

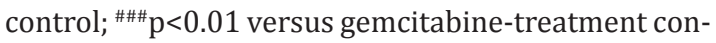
trol group. (D and E) Cell proliferation was examined by colony formation assay. Data are presented as the mean \pm SD. ${ }^{* * *} \mathrm{p}<0.001$ versus the control; ${ }^{\# \# \# p<0.01}$ versus gemcitabine-treatment control group. ( $\mathrm{F}$ and G) Apoptotic cells were detected by Annexin V-PI double staining. (H) Representative images of immunofluorescence staining for LC3. (I-P) Western blot analysis of autophagy (I-L) and apoptosis (M-P) related proteins. Data are presented as the mean \pm SD. ${ }^{*} \mathrm{p}<0.05,{ }^{* * *} \mathrm{p}<0.001$ versus the control; ${ }^{\# \# \#} \mathrm{p}<0.01$ versus gemcitabine-treatment control group.

in parallel with the downregulation of USP22 in response to miR-29c overexpression (Fig. $1 \mathrm{P}$ and $1 \mathrm{Q})$.

USP22 knockdown inhibits growth and promotes apoptosis by inducing autophagy

The mechanisms regulating autophagy and apoptosis in PC were examined by

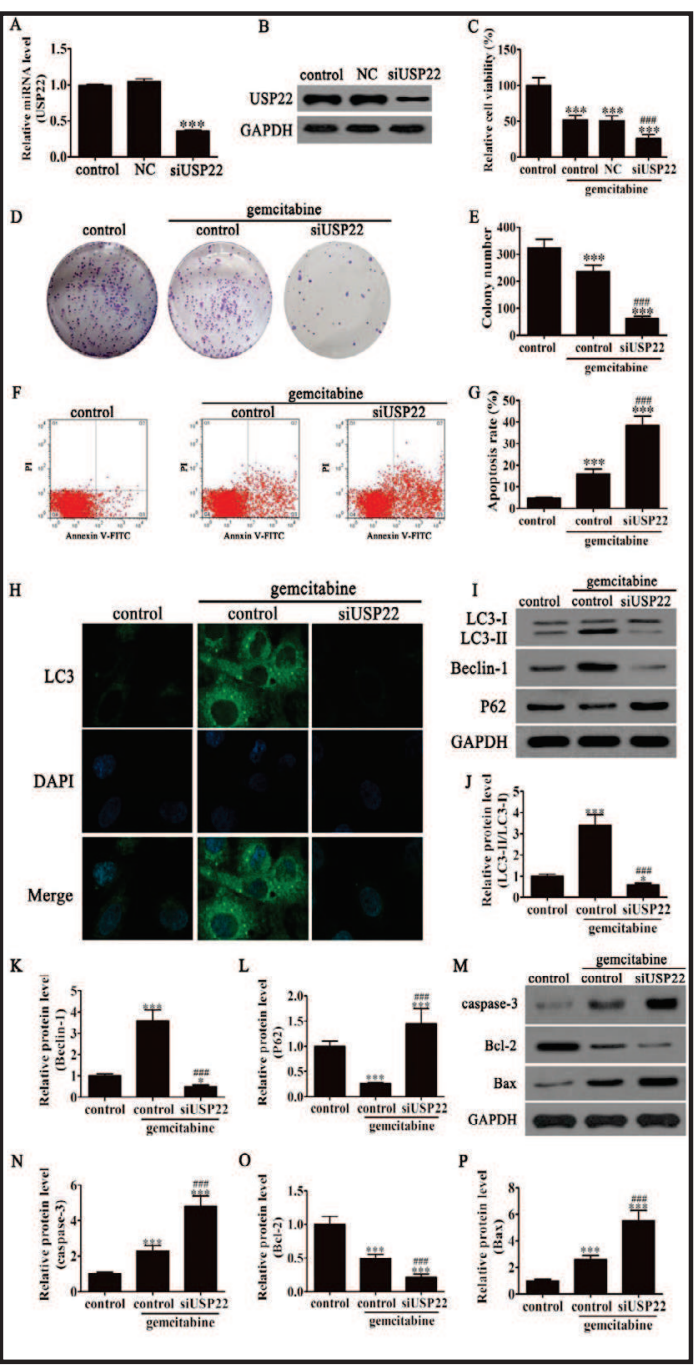
silencing USP22, which is overexpressed in PC tissue samples and suggested to play an oncogenic role [35]. Fig. 2A shows the effective siRNA-mediated downregulation of USP22, which resulted in an approximately $75 \%$ decrease in USP22 expression compared with that in the NC control in Panc-1 cells. USP22 silencing significantly enhanced the gemcitabine-induced decrease in cell viability and colony formation (Fig. 2C-E). Flow cytometric analysis showed that transfection with siUSP22 resulted in an approximately 2.5 -fold increase in gemcitabine-induced apoptosis (Fig. 2F and G). Western blot analysis showed that siUSP22 enhanced the gemcitabineinduced upregulation of caspase- 3 and Bax and downregulation of Bcl-2, suggesting that USP22 inhibits gemcitabine-induced apoptosis (Fig. 2M-P). Immunofluorescence analysis showed that USP22 silencing abolished the gemcitabine-induced upregulation of LC3, and western blot analysis showed that siUSP22 suppressed the gemcitabine-induced increase in the LC3-II/LC3-1 ratio, the upregulation of Beclin-1, and downregulation of P62, suggesting that USP22 promotes autophagy in response to gemcitabine in Panc-1 cells (Fig. 2H-L).

\section{USP22 is a target of miR-29c}

To determine whether USP22 is a target of miR-29c, the miRanda algorithm was searched for complementary sequences between miR-29c and the 3'-UTR of USP22. The sequence alignment and identification of complementary sequences are shown in Fig. 3A. The wild-type and mutant USP22 3'-UTR sequences were fused to aluciferase reportervector and transfected together with miR-29c mimics into HEK293T cells to confirm that USP22 is the target of 


\section{Cellular Physiology Cell Physiol Biochem 2018;47:747-758 \begin{tabular}{c|c} 
DOI: 10.1159/000490027 & O 2018 The Author(s). Published by S. Karger AG, Basel \\
wwww
\end{tabular} \\ Huang et al.: Inhibition of USP22-Mediated Autophagy by MIR-29c Increases Chemosensitivity}

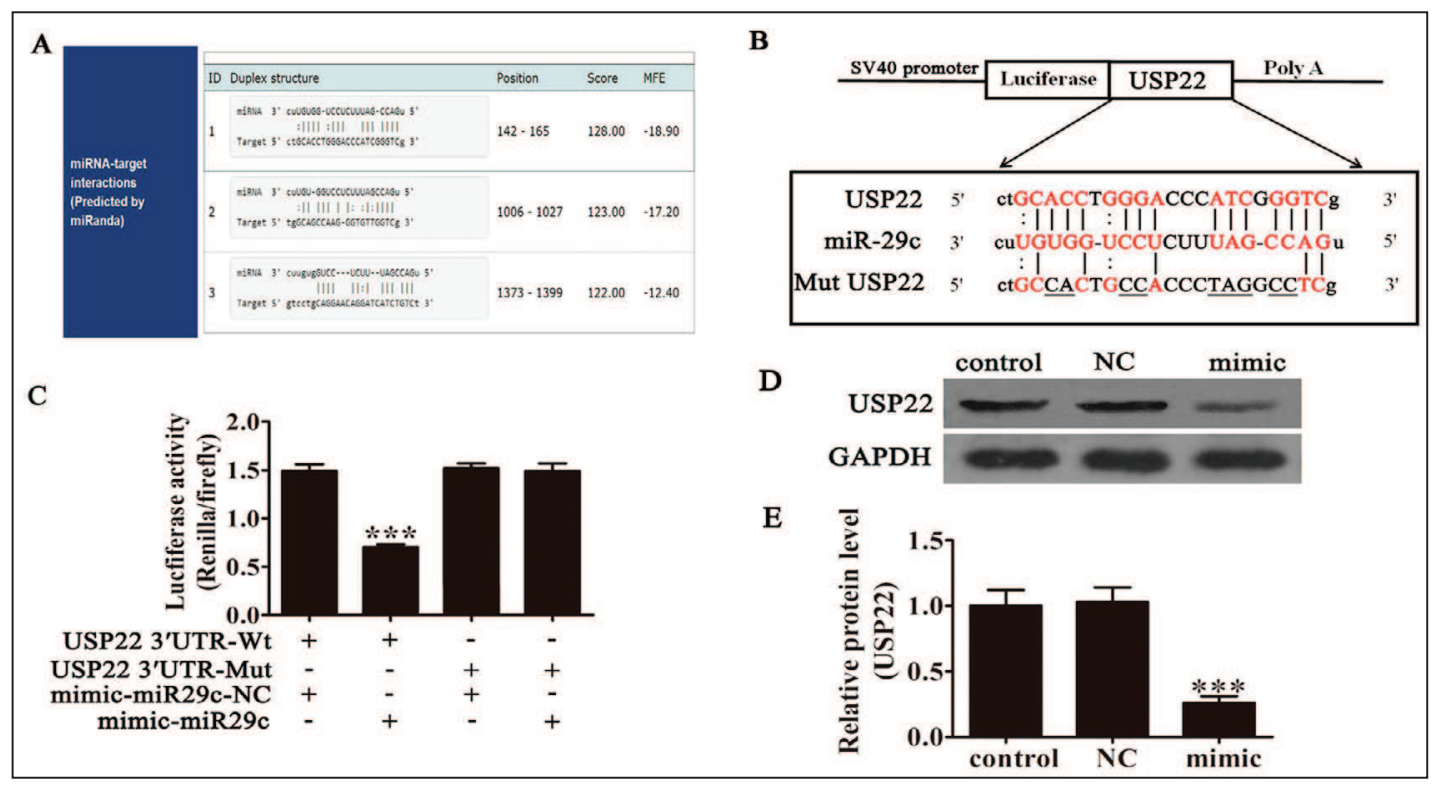

Fig. 3. USP 22 is a target of miR-29c. (A, B) Complementary sequences between miR-29c and the $3^{\prime}$-UTR of USP22 mRNA were obtained using publicly available algorithms. The mutated version of the USP22 $3^{\prime}$ UTR is also shown. (C) The wild-type or mutant 3'-UTR of USP22 was fused to the luciferase coding region (PYr-RGS-17 3'-UTR) and co-transfected into HEK293T cells with miR-29c mimics or miR-29c-NC, and the relative luciferase activity was determined at $48 \mathrm{~h}$ after transfection. The data are presented as the mean $\pm \mathrm{SD} .{ }^{* * *} \mathrm{p}<0.001$ versus the control. (D and E) Western blot analysis of USP22 expression in Panc- 1 cells transfected with miR-29c mimics or miR-NC. $n=5$. GAPDH expression levels were detected as an endogenous control. The data are presented as the mean \pm SD. ${ }^{* * *} \mathrm{p}<0.001$ versus the control.

miR-29c. A luciferase activity assay showed that miR-29c overexpression inhibited the activity of the wild-type but not that of the mutant USP22 $3^{\prime}$ UTR (Fig. 3C). Western blot analysis showed that miR-29c overexpression downregulated USP22 expression in Panc-1 cells (Fig. 3D and 3E).

miR-29c promotes $P C$ cell chemosensitivity by inducing of apoptosis via the inhibition of autophagy

To examine the mechanism underlying the effect of miR-29c on the chemosensitivity of PC cells and the involvement of autophagy, cells transfected with miR-29c mimics were pretreated with the

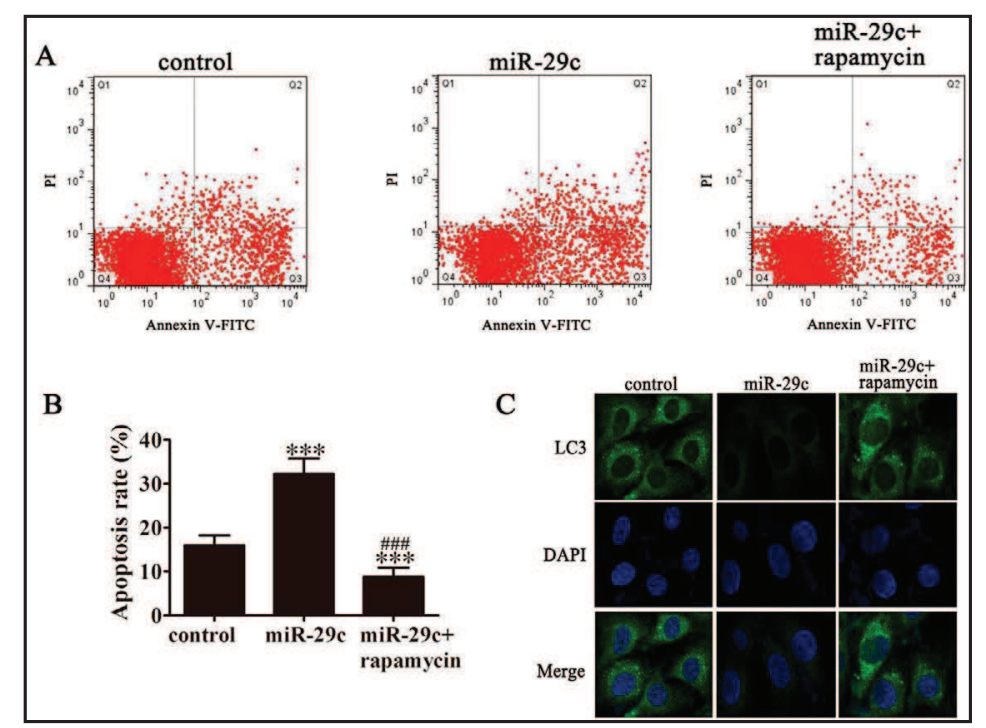

Fig. 4. miR-29c induces apoptosis by inhibiting autophagy activation. Panc-1 cells transfected with miR-29c were pretreated with the autophagy agonist rapamycin for $6 \mathrm{~h}$, followed by exposure to $100 \mathrm{ng} / \mathrm{ml}$ gemcitabine for $24 \mathrm{~h}$. (A and B) Apoptosis was examined by Annexin VFITC and flow cytometry and the apoptotic rate was calculated. Data are presented as the mean $\pm \mathrm{SD}$. ${ }^{* * *} \mathrm{p}<0.001$ versus the control. ${ }^{\# \# \#} \mathrm{p}<0.01$ versus miR-29c overexpression group. (C) Representative images of immunofluorescence staining for LC3. 


\section{Cellular Physiology Cell Physiol Biochem 2018;47:747-758 \begin{tabular}{c|c} 
DOI: 10.1159/000490027 & O 2018 The Author(s). Published by S. Karger AG, Basel \\
and Biochemistry Published online: Nay 28, 2018 & www
\end{tabular} Huang et al.: Inhibition of USP22-Mediated Autophagy by MIR-29c Increases Chemosensitivity}

autophagy inducer rapamycin before treatment with gemcitabine. Flow cytometric analysis showed that rapamycin significantly inhibited miR-29c induced apoptosis (Fig. 4A and B) and abolished miR-29c mediated inhibition of autophagy (Fig. 4C), confirming that miR-29c promotes the chemosensitivity of PC cells by inhibiting autophagy and inducing apoptosis.

miR-29c targets USP22 and suppresses autophagy-mediated chemoresistance in a xenograft tumor model in vivo

The role of miR29c in the resistance of PC to gemcitabine was investigated in vivo in a xenograft mouse model established from miR-29c overexpressing or control cells followed by treatment with or without gemcitabine. Tumor growth was monitored for 4 weeks. The results showed that gemcitabine inhibited tumor growth and this effect was increased significantly by miR-29c overexpression (Fig. 5A and B). Gemcitabine downregulated miR29c expression by 50\%, whereas this effect was abolished by miR-29c mimic treatment, which upregulated miR-29c by approximately 3.5-fold, and miR-29c overexpression

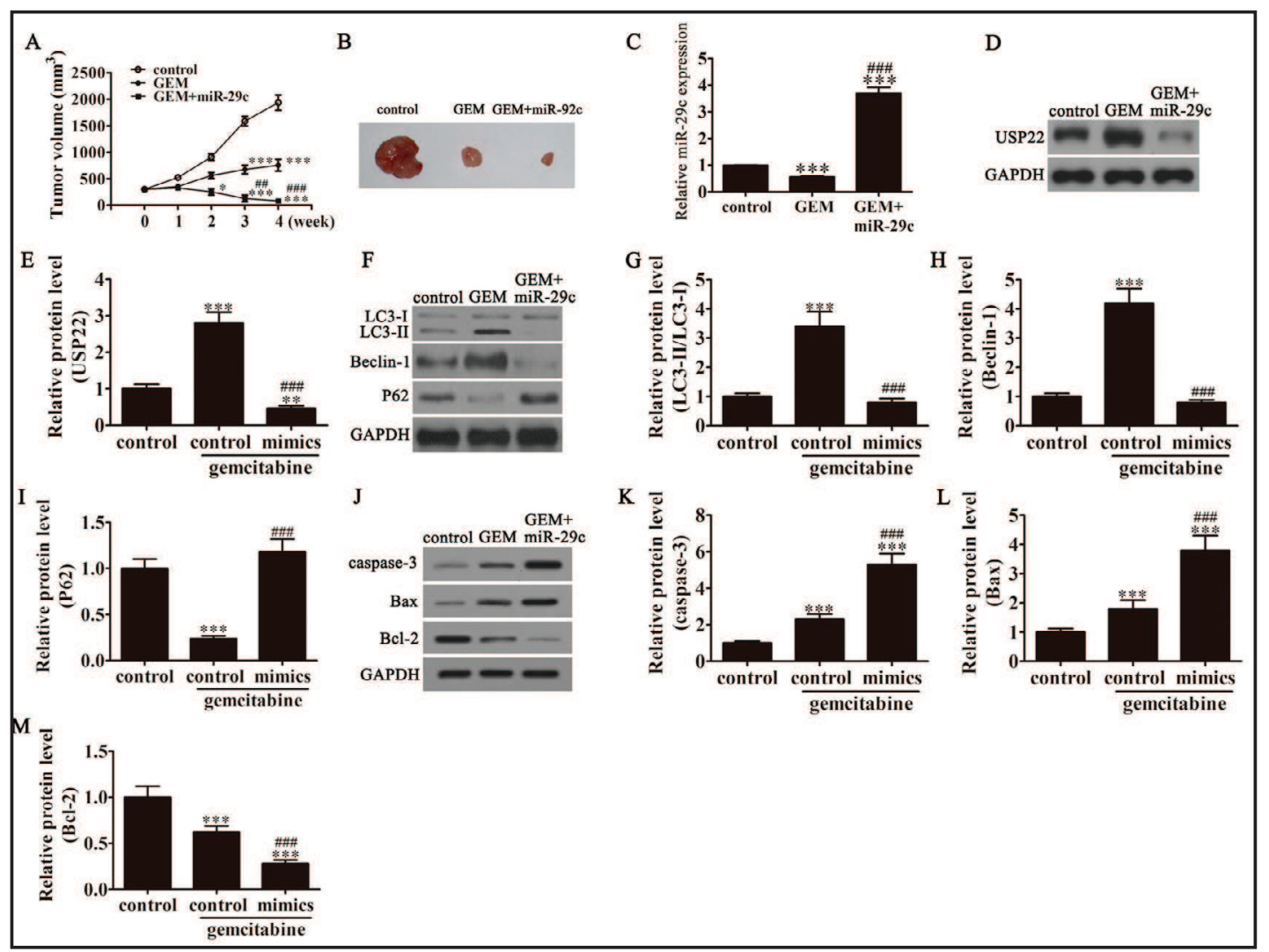

Fig. 5. miR-29c targets USP22 and suppresses autophagy-mediated chemoresistance in a xenograft model. A xenograft mouse model established from miR-29c overexpressing or control cells. When the tumor volume reached $300 \mathrm{~mm}^{3}$, gemcitabine (GEM) was given intraperitoneally to mice at a dose of $50 \mathrm{mg} / \mathrm{kg}$ once a week for 4 weeks. (A) Growth curves showing xenograft tumor volumes measured once a week for 4 weeks. $\mathrm{n}=6$. ${ }^{*} \mathrm{p}<0.05 ;{ }^{* * *} \mathrm{p}<0.001$ versus control group; ${ }^{\# \#} \mathrm{p}<0.01$ versus miR-29c overexpression group. (B) Photographs of tumor tissues in the different groups on day 30. (C) miR-29c expression was detected by qRt-PCR. $\mathrm{n}=6$. Data are presented as the mean \pm SD. ${ }^{* * *} \mathrm{p}<0.001$ versus control group; ${ }^{\# \# \#} \mathrm{p}<0.01$ versus miR-29c over expression group. (D and E) Western blot analysis of USP22 expression. Data are presented as the mean $\pm \mathrm{SD} .{ }^{* *} \mathrm{p}<0.01,{ }^{* * *} \mathrm{p}<0.001$ versus control group; ${ }^{\# \#} \mathrm{p}<0.01$ versus miR-29c over expression group. (F-M) Western blot analysis of autophagy (F-I) and apoptosis (J-M) related proteins. Data are presented as the mean \pm SD. ${ }^{* * *} \mathrm{p}<0.001$ versus control group; ${ }^{\# \#} \mathrm{p}<0.01$ versus miR-29c over expression group. 


\section{Cellular Physiology Cell Physiol Biochem 2018;47:747-758 \\ \begin{tabular}{l|l} 
and Biochemistry $10.1159 / 000490027$ & $\begin{array}{l}\text { D) } 2018 \text { The Author(s). Published by S. Karger AG, Base } \\
\text { www.karger.com/cpb }\end{array}$ \\
\cline { 2 - 4 }
\end{tabular} \\ Huang et al.: Inhibition of USP22-Mediated Autophagy by MIR-29c Increases Chemosensitivity}

markedly downregulated USP22 protein expression in mice (Fig. 5C-E). This occurred in parallel with a decrease in the LC3-II/LC3-1 ratio, the downregulation of Beclin-1, and the upregulation of p62, indicating the inhibition of autophagy (Fig. 5F-I). Western blot analysis of apoptosis related proteins showed that apoptosis was induced in miR-29c overexpressing tumors treated with gemcitabine, as indicated by the upregulation of caspase- 3 and Bax and the downregulation of Bcl-2 (Fig. 5J-M). Taken together, these results indicated that miR-29c increases the chemosensitivity of PC xenograft tumors by downregulating USP22, leading to the inhibition of autophagy and induction of apoptosis.

\section{Discussion}

The maintenance of homeostasis requires a balance between apoptosis and cell proliferation, and pancreatic cancer cells have the capacity to disrupt cellular homeostasis to promote malignant growth [40]. Autophagy, which plays an important role in cell homeostasis, can promote or suppress tumorigenesis, and PC cells are characterized by increased autophagy and a dependence on autophagy for growth. In the present study, we show that USP22 plays a role in the chemoresistance of PC cells by inducing autophagy, leading to the inhibition of gemcitabine-induced apoptosis, and miR-29c has the opposite effects, promoting chemosensitivity by downregulating USP22.

Deubiquitinating enzymes (DUBs) play important roles in cell homeostasis, and deregulation of DUBs is associated with cancer through the modulation of p53, Wnt, EGFR, and NF- $\kappa B$ pathways, affecting cell cycle progression and apoptosis [41]. USP22 is a highly conserved DUB that functions in the regulation of the cell cycle, and its overexpression is associated with tumor progression in several types of cancer [42, 43]. USP22 knockdown leads to cell cycle arrest at the G0/G1 phase and decreased tumor growth, indicating that USP22 plays an oncogenic role [32, 44, 45]. Although USP22 is considered a potential target for cancer therapy, the regulation of its expression has not been elucidated in detail. USP22 protein levels are increased by c-Myc, whereas the USP22 substrate SIRT1 deacetylates USP2 2 and inactivates it $[32,45,46]$. USP22 activity is also regulated by acetylation on lysine 129 as well as other post-transcriptional modifications such as phosphorylation by cyclindependent kinase 1, which activates USP22 [41]. Here, we showed that USP22 is a direct target of miR-29c, which downregulates USP22 expression in PC.

In the present study, miR-29c overexpression increased the effect of gemcitabine on inhibiting PC cell viability and colony formation and promoting apoptosis, indicating that miR$29 \mathrm{c}$ increases the chemosensitivity of PC cells. miR-29c was previously shown to suppress invasion and metastasis in PC by downregulating the expression of matrix metalloprotease 2 [47]. miR-29c is downregulated in PC tissues and cell lines, and its overexpression inhibits PC cell proliferation, migration and invasion by targeting the ITGB1 gene, which encodes the beta 1 integrin CD29 [48]. miR-29c downregulation increases the migration and stem cell-like phenotype of PC cells [20]. We specifically investigated the role of miR-29c in the chemoresistance of PC cells and showed that miR-29c enhanced the effect of gemcitabine by suppressing gemcitabine-induced autophagy, suggesting that the chemosensitizing effect of miR-29c involves the modulation of autophagy.

Because the effect of miR-29c occurred in parallel with the downregulation of USP22, we investigated the involvement of the miR-29c/USP22 axis on the chemosensitivity of PC cells. USP22 silencing experiments indicated that downregulation of USP22 decreases PC cell chemoresistance by suppressing autophagy and promoting apoptosis. The role of autophagy in cancer is complex, although evidence suggests that autophagy promotes cancer cell survival [49]. Activation of autophagy in PC is associated with poor survival, and inhibition of autophagy suppresses PC cell growth in vitro and tumor growth in vivo [50], supporting the notion that autophagy is necessary for PC progression. The present results showing that miR-29c suppresses the proliferation and promotes the chemosensitivity of pancreatic cancer cells by targeting USP22-mediated autophagy were consistent with previous studies. 


\section{Cellular Physiology Cell Physiol Biochem 2018;47:747-758 \begin{tabular}{c|l}
\hline DOI: 10.1159/000490027 & @ 2018 The Author(s). Published by S. Karger AG, Basel
\end{tabular} and Biochemistry

Inhibition of autophagy was shown to increase the anti-tumor effect of gemcitabine in PC cells [51]. The role of autophagy in the resistance of tumor cells to gemcitabine was also demonstrated in other cancers. In lung cancer, autophagy contributes to chemoresistance by protecting cells from gemcitabine-induced apoptosis [52]. Similarly, in triple-negative breast cancer, autophagy protects cells from gemcitabine-induced apoptosis, and inhibition of autophagy promotes apoptosis [53]. These studies support the present results showing that USP22 inhibits gemcitabine-induced apoptosis and increases PC chemoresistance. In the present study, we elucidated a potential mechanism underlying PC chemoresistance by which miR-29c increases PC chemosensitivity by targeting USP22 mediated autophagy. These findings were confirmed in vivo in a xenograft mouse model in which miR-29c increased the effect of gemcitabine on decreasing tumor growth by inhibiting autophagy and inducing apoptosis.

In conclusion, a series of gain and loss of function experiments showed that miR29c promotes chemosensitivity in PC by inhibiting autophagy. We showed that miR-29c directly targets and downregulates USP22, increasing chemosensitivity by inhibiting USP22-mediated autophagy and inducing apoptosis in vitro and in vivo. These results elucidate a novel pathway of USP 22 regulation and suggest a potential strategy to reduce the chemoresistance of PC cells.

\section{Acknowledgements}

This study was supported by the National Natural Science Foundation of China (Grant no. 81660451); The Science and Technology Project of Guiyang municipal, Guizhou province (Grant no. 2014100162); The Science and technology Department of Guizhou province (Grant no. 20152088, 20147017).

\section{Disclosure Statement}

The authors declare no conflict of interests.

\section{References}

-1 DeSantis CE, Lin CC, Mariotto AB, Siegel RL, Stein KD, Kramer JL, Alteri R, Robbins AS, Jemal A: Cancer treatment and survivorship statistics, 2014. CA Cancer J Clin 2014;64:252-271.

2 Klein AP: Genetic susceptibility to pancreatic cancer. Mol Carcinog 2012;51:14-24.

3 Huang C, Xie K: Analysis of the potential for pancreatic cancer metastasis in vitro and in vivo. Methods Mol Biol 2013;980:301-319.

4 Siegel RL, Fedewa SA, Miller KD, Goding-Sauer A, Pinheiro PS, Martinez-Tyson D, Jemal A: Cancer statistics for hispanics/latinos, 2015. CA Cancer J Clin 2015;65:457-480.

-5 Gnanamony M, Gondi CS: Chemoresistance in pancreatic cancer: Emerging concepts. Oncol Lett 2017;13:2507-2513.

6 Xiao G, Wang X, Yu Y: Cxcr4/let-7a axis regulates metastasis and chemoresistance of pancreatic cancer cells through targeting hmga2. Cell Physiol Biochem 2017;43:840-851.

-7 Rossi ML, Rehman AA, Gondi CS: Therapeutic options for the management of pancreatic cancer. World J Gastroenterol 2014;20:11142-11159.

8 de Sousa Cavalcante L, Monteiro G: Gemcitabine: Metabolism and molecular mechanisms of action, sensitivity and chemoresistance in pancreatic cancer. Eur J Pharmacol 2014;741:8-16.

-9 Ma C, Nong K, Wu B, Dong B, Bai Y, Zhu H, Wang W, Huang X, Yuan Z, Ai K: Mir-212 promotes pancreatic cancer cell growth and invasion by targeting the hedgehog signaling pathway receptor patched-1. J Exp Clin Cancer Res 2014;33:54. 


\section{Cellular Physiology Cell Physiol Biochem 2018;47:747-758

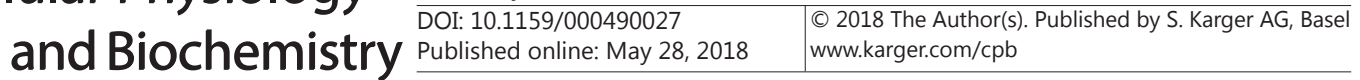 \\ Huang et al.: Inhibition of USP22-Mediated Autophagy by MIR-29c Increases Chemosensitivity}

10 Wang D, Qiu C, Zhang H, Wang J, Cui Q, Yin Y: Human microrna oncogenes and tumor suppressors show significantly different biological patterns: From functions to targets. PLoS One 2010;5

11 Esquela-Kerscher A, Slack FJ: Oncomirs - micrornas with a role in cancer. Nat Rev Cancer 2006;6:259-269.

12 Qian Y, Feng L, Wu W, Weng T, Hu C, Hong B, Wang FXC, Shen L, Wang Q Jin X, Yao H: Microrna expression profiling of pancreatic cancer cell line 13.6p1 following b7-h4 knockdown. Cell Physiol Biochem 2017;44:494-504.

13 Li H, Hao X, Wang H, Liu Z, He Y, Pu M, Zhang H, Yu H, Duan J, Qu S: Circular rna expression profile of pancreatic ductal adenocarcinoma revealed by microarray. Cell Physiol Biochem 2016;40:1334-1344.

14 Deng T, Yuan Y, Zhang C, Yao W, Wang C, Liu R, Ba Y: Identification of circulating mir-25 as a potential biomarker for pancreatic cancer diagnosis. Cell Physiol Biochem 2016;39:1716-1722.

15 Pai P, Rachagani S, Are C, Batra SK: Prospects of mirna-based therapy for pancreatic cancer. Curr Drug Targets 2013;14:1101-1109.

-16 Frampton AE, Fletcher CE, Gall TM, Castellano L, Bevan CL, Stebbing J, Krell J: Circulating peripheral blood mononuclear cells exhibit altered mirna expression patterns in pancreatic cancer. Expert Rev Mol Diagn 2013;13:425-430.

17 Humeau M, Torrisani J, Cordelier P: Mirna in clinical practice: Pancreatic cancer. Clin Biochem 2013;46:933-936.

18 Huang F, Tang J, Zhuang X, Zhuang Y, Cheng W, Chen W, Yao H, Zhang S: Mir-196a promotes pancreatic cancer progression by targeting nuclear factor kappa-b-inhibitor alpha. PLoS One 2014;9:e87897.

-19 Sicard F, Gayral M, Lulka H, Buscail L, Cordelier P: Targeting mir-21 for the therapy of pancreatic cancer. Mol Ther 2013;21:986-994.

20 Jiang J, Yu C, Chen M, Zhang H, Tian S, Sun C: Reduction of mir-29c enhances pancreatic cancer cell migration and stem cell-like phenotype. Oncotarget 2015;6:2767-2778.

21 Bae HJ, Noh JH, Kim JK, Eun JW, Jung KH, Kim MG, Chang YG, Shen Q, Kim SJ, Park WS, Lee JY, Nam SW: Microrna-29c functions as a tumor suppressor by direct targeting oncogenic sirt1 in hepatocellular carcinoma. Oncogene 2014;33:2557-2567.

-22 Liu N, Tang LL, Sun Y, Cui RX, Wang HY, Huang BJ, He QM, Jiang W, Ma J: Mir-29c suppresses invasion and metastasis by targeting tiam1 in nasopharyngeal carcinoma. Cancer Lett 2013;329:181-188.

23 Fan YC, Mei PJ, Chen C, Miao FA, Zhang H, Li ZL: Mir-29c inhibits glioma cell proliferation, migration, invasion and angiogenesis. J Neurooncol 2013;115:179-188.

24 Han TS, Hur K, Xu G, Choi B, Okugawa Y, Toiyama Y, Oshima H, Oshima M, Lee HJ, Kim VN, Chang AN, Goel A, Yang HK: Microrna-29c mediates initiation of gastric carcinogenesis by directly targeting itgb1. Gut 2015;64:203-214.

-25 Fan Y, Song X, Du H, Luo C, Wang X, Yang X, Wang Y, Wu X: Down-regulation of mir-29c in human bladder cancer and the inhibition of proliferation in $t 24$ cell via pi3k-akt pathway. Med Oncol 2014;31:65.

-26 Zhang JX, Mai SJ, Huang XX, Wang FW, Liao YJ, Lin MC, Kung HF, Zeng YX, Xie D: Mir-29c mediates epithelialto-mesenchymal transition in human colorectal carcinoma metastasis via ptp4a and gna13 regulation of beta-catenin signaling. Ann Oncol 2014;25:2196-2204.

27 Hong Q Fang J, Pang Y, Zheng J: Prognostic value of the microrna-29 family in patients with primary osteosarcomas. Med Oncol 2014;31:37.

28 Hoeller D, Dikic I: Targeting the ubiquitin system in cancer therapy. Nature 2009;458:438-444.

29 Zhang Y, Yao L, Zhang X, Ji H, Wang L, Sun S, Pang D: Elevated expression of usp22 in correlation with poor prognosis in patients with invasive breast cancer. J Cancer Res Clin Oncol 2011;137:1245-1253.

-30 Liu YL, Yang YM, Xu H, Dong XS: Increased expression of ubiquitin-specific protease 22 can promote cancer progression and predict therapy failure in human colorectal cancer. J Gastroenterol Hepatol 2010;25:18001805.

-31 Wang H, Li YP, Chen JH, Yuan SF, Wang L, Zhang JL, Yao Q, Li NL, Bian JF, Fan J, Yi J, Ling R: Prognostic significance of usp22 as an oncogene in papillary thyroid carcinoma. Tumour Biol 2013;34:1635-1639.

32 Lv L, Xiao XY, Gu ZH, Zeng FQ, Huang LQ, Jiang GS: Silencing usp22 by asymmetric structure of interfering rna inhibits proliferation and induces cell cycle arrest in bladder cancer cells. Mol Cell Biochem 2011;346:11-21.

-33 Li ZH, Yu Y, Du C, Fu H, Wang J, Tian Y: Rna interference-mediated usp22 gene silencing promotes human brain glioma apoptosis and induces cell cycle arrest. Oncol Lett 2013;5:1290-1294.

-34 Zhao HD, Tang HL, Liu NN, Zhao YL, Liu QQ, Zhu XS, Jia LT, Gao CF, Yang AG, Li JT: Targeting ubiquitin- 


\section{Cellular Physiology Cell Physiol Biochem 2018;47:747-758 \begin{tabular}{c|c|c|c|}
\cline { 2 - 3 } DOI: 10.1159/000490027 & (c) 2018 The Author(s). Published by S. Karger AG, Basel
\end{tabular} and Biochemistry Published online: May 28, 2018 www.karger.com/cpb \\ Huang et al.: Inhibition of USP22-Mediated Autophagy by MIR-29c Increases Chemosensitivity}

specific protease 22 suppresses growth and metastasis of anaplastic thyroid carcinoma. Oncotarget 2016;7:31191-31203.

-35 Liang JX, Ning Z, Gao W, Ling J, Wang AM, Luo HF, Liang Y, Yan Q, Wang ZY: Ubiquitinspecific protease 22induced autophagy is correlated with poor prognosis of pancreatic cancer. Oncol Rep 2014;32:27262734.

36 Ogier-Denis E, Codogno P: Autophagy: A barrier or an adaptive response to cancer. Biochim Biophys Acta 2003;1603:113-128.

37 Tanida I, Ueno T, Kominami E: Lc3 and autophagy. Methods Mol Biol 2008;445:77-88.

-38 Mathew R, Karantza-Wadsworth V, White E: Role of autophagy in cancer. Nat Rev Cancer 2007;7:961-967.

- 39 Liu F, Liu D, Yang Y, Zhao S: Effect of autophagy inhibition on chemotherapy-induced apoptosis in a549 lung cancer cells. Oncol Lett 2013;5:1261-1265.

40 New M, Van Acker T, Long JS, Sakamaki JI, Ryan KM, Tooze SA: Molecular pathways controlling autophagy in pancreatic cancer. Front Oncol 2017;7:28.

41 Melo-Cardenas J, Zhang Y, Zhang DD, Fang D: Ubiquitin-specific peptidase 22 functions and its involvement in disease. Oncotarget 2016;7:44848-44856.

42 Piao S, Liu Y, Hu J, Guo F, Ma J, Sun Y, Zhang B: Usp22 is useful as a novel molecular marker for predicting disease progression and patient prognosis of oral squamous cell carcinoma. PLoS One 2012;7:e42540.

43 Tang B, Tang F, Li B, Yuan S, Xu Q, Tomlinson S, Jin J, Hu W, He S: High usp22 expression indicates poor prognosis in hepatocellular carcinoma. Oncotarget 2015;6:12654-12667.

-44 Zhang XY, Varthi M, Sykes SM, Phillips C, Warzecha C, Zhu W, Wyce A, Thorne AW, Berger SL, McMahon SB: The putative cancer stem cell marker usp22 is a subunit of the human saga complex required for activated transcription and cell-cycle progression. Mol Cell 2008;29:102-111.

-45 Li L, Osdal T, Ho Y, Chun S, McDonald T, Agarwal P, Lin A, Chu S, Qi J, Hsieh YT, Dos Santos C, Yuan H, Ha TQ, Popa M, Hovland R, Bruserud O, Gjertsen BT, Kuo YH, Chen W, Lain S, McCormack E, Bhatia R: Sirt1 activation by a c-myc oncogenic network promotes the maintenance and drug resistance of human flt3-itd acute myeloid leukemia stem cells. Cell Stem Cell 2014;15:431-446.

46 Armour SM, Bennett EJ, Braun CR, Zhang XY, McMahon SB, Gygi SP, Harper JW, Sinclair DA: A highconfidence interaction map identifies sirt1 as a mediator of acetylation of usp22 and the saga coactivator complex. Mol Cell Biol 2013;33:1487-1502.

47 Zou Y, Li J, Chen Z, Li X, Zheng S, Yi D, Zhong A, Chen J: Mir-29c suppresses pancreatic cancer liver metastasis in an orthotopic implantation model in nude mice and affects survival in pancreatic cancer patients. Carcinogenesis 2015;36:676-684.

48 Lu Y, Hu J, Sun W, Li S, Deng S, Li M: Mir-29c inhibits cell growth, invasion, and migration of pancreatic cancer by targeting itgb1. Onco Targets Ther 2016;9:99-109.

-49 White E, DiPaola RS: The double-edged sword of autophagy modulation in cancer. Clin Cancer Res 2009;15:5308-5316.

-50 Yang S, Wang X, Contino G, Liesa M, Sahin E, Ying H, Bause A, Li Y, Stommel JM, Dell'antonio G, Mautner J, Tonon G, Haigis M, Shirihai OS, Doglioni C, Bardeesy N, Kimmelman AC: Pancreatic cancers require autophagy for tumor growth. Genes Dev 2011;25:717-729.

-51 Yang MC, Wang HC, Hou YC, Tung HL, Chiu TJ, Shan YS: Blockade of autophagy reduces pancreatic cancer stem cell activity and potentiates the tumoricidal effect of gemcitabine. Mol Cancer 2015;14:179.

52 Wu HM, Shao LJ, Jiang ZF, Liu RY: Gemcitabine-induced autophagy protects human lung cancer cells from apoptotic death. Lung 2016;194:959-966.

53 Chen M, He M, Song Y, Chen L, Xiao P, Wan X, Dai F, Shen P: The cytoprotective role of gemcitabine-induced autophagy associated with apoptosis inhibition in triple-negative mda-mb-231 breast cancer cells. Int J Mol Med 2014;34:276-282. 